\title{
Dynamical Model Averaging and PWM Based Control for Pneumatic Actuators
}

\author{
Sean Hodgson ${ }^{1}$, Mahdi Tavakoli ${ }^{1}$, Minh Tu Pham ${ }^{2}$, Arnaud Lelevé ${ }^{2}$
}

\begin{abstract}
A pneumatic actuator with solenoid valves is a discontinuous-input system because each valve can be either in on or off state. For such an actuator, this paper proposes a sliding-mode control scheme based on an averaged continuousinput equivalent model for the open-loop system. The averaged model is obtained from the nonlinear dynamics of the open-loop discontinuous-input system undergoing pulse-width-modulation (PWM) at the input (i.e., valve open/close action). The PWM duty cycle will be regarded as a continuous input to the proposed averaged model, and thus generated by the proposed sliding-mode controller. By adjusting the PWM duty cycle, the controller switches between seven modes of operation of the open-loop system in order to select the ones with necessary and sufficient amounts of drive energy to achieve position tracking. We will show that this results in reduced position error and valve switching activity for the actuator. The proposed control scheme is experimentally used in the position control of a pneumatic actuator and the results are presented.
\end{abstract}

Index Terms-Pneumatic actuator, on/off solenoid valve, PWM, sliding-mode control design, position tracking.

\section{INTRODUCTION}

In this study, we investigate the control of pneumatic actuators used in robots. Pneumatic actuators are widely used in many different industries and offer many advantages such as low cost, good power/weight ratio, cleanliness, and safety [1]. However, they suffer from drawbacks including friction and variation of the actuator dynamics to load and piston position along the cylinder stroke [2]. Controlling the position of a pneumatic actuator is difficult due to the nonlinear dynamics of the system [3].

For excitation, some pneumatic systems are equipped with on/off solenoid valves. These valves represent a low-cost alternative to servo-valves, which allow for continuous control of the input mass flow rate. The difficulty in utilizing on/off solenoid valves is that precise control of the piston position will be hampered by the discrete-input (on/off) nature of these valves. This makes the actuator position control even more difficult.

If the nonlinear dynamics of a pneumatic system can be approximated as an equivalent linear system, then linear controllers can be designed [1]. However, it is preferable

\footnotetext{
1 with Department of Electrical and Computer Engineering, University of Alberta, Edmonton, AB T6G2V4, Canada mahdi.tavakoli@ualberta.ca

2 with Laboratoire Ampère, UMR CNRS 5005, Université de Lyon, INSA-LYON, F-69621 Villeurbanne Cedex, France minh-tu.phameinsa-lyon.fr
}

to design controllers that take into account the open-loop system nonlinearities. PWM controllers have been developed in the past to control the position of solenoid-valve pneumatic actuators [4], [5], [6], [7]. A PWM input with a high switching frequency approximates a continuous input whose magnitude is determined by the PWM duty cycle. This is advantageous because it allows us to provide to a discreteinput system what is effectively a continuous input.

On the other hand, it is possible to employ nonlinear sliding-mode control for solenoid-valve systems [8]. Slidingmode control alters the dynamics of a system by the application of a high-frequency switching control [9], [10]. Sliding-mode control is parametrically very robust and can account for dynamic uncertainties as well as nonlinearities of a system. While using pneumatic actuators equipped with solenoid-valve it turns out that these systems are by essence nonlinear with discontinuous-time dynamics. To control such systems one can either use hybrid control theory or construct an equivalent continuous-time dynamics of the system then use nonlinear control theory. In [11], nonlinear model averaging is combined with PWM and sliding-mode control to control a solenoid-valve pneumatic actuator based on a three mode model.

In our previous works, a sliding mode control based on a seven mode switching law has been proposed [12]. It has been shown in these works that four additional modes allow not only to decrease the coarseness of the drive force for lower position tracking errors but also to reduce the number of switching. The current paper investigates a PWM switching law, provided by a sliding mode controller, which is designed from an averaged model of the system. In [11], an approach based on a three mode model has been introduced, in the current paper we provide a theoretical extension from three modes to seven modes. Our proposal is supported by experimental results and a comparison study with another controller. Moreover robustness with respect to load variations and input excitations are given to assess the performances of the controller.

The organization of this paper is as follows. The discrete input model of the actuator is found in Section II. A nonlinear averaged continuous-input model of the open-loop actuator is obtained in Section III. A sliding-mode control for the pneumatic actuator is proposed in Section IV. The experimental results validating the proposed controllers are shown in Section V. Finally, the concluding remarks are 


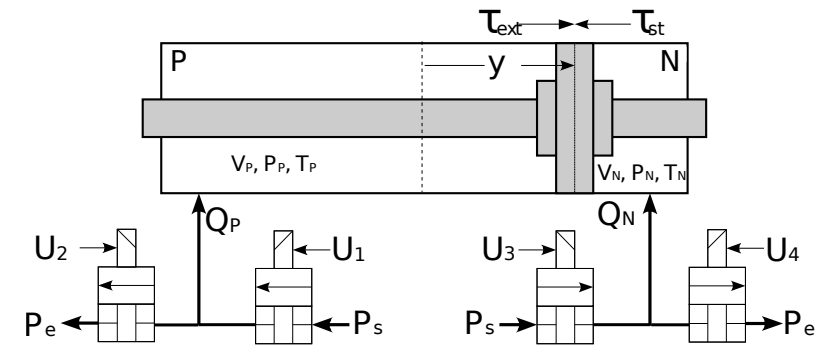

Fig. 1. A pneumatic actuator with 4 on/off solenoid valves.

presented in Section VI.

\section{DISCRETE-INPUT MODEL OF THE OPEN-LOOP ACTUATOR}

The modelling of a pneumatic actuator with its chamber and four solenoid valves is visible in [12]. It is possible to write the dynamics of the open-loop pneumatic actuator in a discrete input form, assuming that the temperature variation in chambers is negligible with respect to the supply temperature (thus $T_{P}=T_{N}=T$ ):

$$
\begin{array}{r}
\dddot{y}=f+\frac{k r T}{M}\left(\frac{Q_{P}}{l / 2+y}-\frac{Q_{N}}{l / 2-y}\right)+\frac{\dot{\tau}_{E x t}}{M} \\
f=\frac{-b_{V}}{M} \ddot{y}-\frac{k}{M}\left(\frac{A_{P} P_{P}}{l / 2+y}+\frac{A_{N} P_{N}}{l / 2-y}\right) \dot{y}
\end{array}
$$

In this paper, we consider a 1-DOF pneumatic actuator comprised of two chambers as shown in Figure 1. Each chamber has two solenoid valves. These solenoid valves allow each chamber to be in one of three states: connected to an air supply (pressurizing), connected to exhaust pressure (venting), or closed (no air flow in or out of chamber). Since each chamber can be in one of the three states of pressurizing, venting or closed, there are a total of nine discrete modes for the two-chamber actuator [13]. These modes are shown in Table I.

TABLE I

Nine Discrete Modes of The Open-Loop Actuator

\begin{tabular}{cccccccc|cc}
\hline \hline & $M_{1}$ & $M_{2}$ & $M_{3}$ & $M_{4}$ & $M_{5}$ & $M_{6}$ & $M_{7}$ & $M_{8}$ & $M_{9}$ \\
\hline$U_{1}$ & 0 & 1 & 0 & 0 & 0 & 1 & 0 & 0 & 1 \\
\hline$U_{2}$ & 0 & 0 & 1 & 0 & 0 & 0 & 1 & 1 & 0 \\
\hline$U_{3}$ & 0 & 0 & 0 & 0 & 1 & 0 & 1 & 0 & 1 \\
\hline$U_{4}$ & 0 & 0 & 0 & 1 & 0 & 1 & 0 & 1 & 0 \\
\hline \hline
\end{tabular}

For each of these nine discrete modes, we obtain the dynamic equation

$$
\dddot{y}= \begin{cases}f+\frac{\dot{\tau}_{E x t}}{M} & , \text { mode } M_{1} \\ f+(-1)^{j} b_{j}+\frac{\dot{\tau}_{E x t}}{M} & , \text { mode } M_{j} \neq M_{1}\end{cases}
$$

where the integer $j$ ranges from 2 to 9 and

$$
\begin{aligned}
b_{2}=\frac{k r T}{M} \frac{Q\left(P_{S}, P_{P}\right)}{(l / 2+y)} & b_{3} & =\frac{k r T}{M} \frac{Q\left(P_{P}, P_{E}\right)}{(l / 2+y)} \\
b_{4}=\frac{k r T}{M} \frac{Q\left(P_{N}, P_{E}\right)}{(l / 2-y)} & b_{5} & =\frac{k r T}{M} \frac{Q\left(P_{S}, P_{N}\right)}{(l / 2-y)} \\
b_{6}=b_{2}+b_{4} & b_{7} & =b_{5}+b_{3} \\
b_{8}=b_{4}-b_{3} & b_{9} & =b_{5}-b_{2}
\end{aligned}
$$

Note that because $P_{E} \leq P_{P} \leq P_{S}, P_{E} \leq P_{N} \leq P_{S}$, $-l / 2 \leq y \leq l / 2$, and mass flow rates are non-negative, functions $b_{2}$ through $b_{7}$ are all positive or equal to zero. Also, $b_{8}$ and $b_{9}$ are approximately equal to 0 and can be positive or negative. As in [12], we will focus on the modes $M_{1}$ to $M_{7}$.

In the next section, we will obtain an averaged continuousinput equivalent for the above discontinuous-input open-loop model. This averaged model is obtained from the nonlinear dynamics of the open-loop system undergoing pulse-width modulation at the input (i.e., valve open/close action). The PWM duty cycle will act as the continuous input to the proposed averaged model.

\section{AVERAGED CONTINUOUS-INPUT MODEL OF THE OPEN-LOOP ACTUATOR}

Let us consider a general dynamic system that may operate in one of $p$ distinct modes at any given time. Within a PWM "period", the system can switch between modes 1 through $p$, due to the variations in the input provided to the system. Switching between the modes 1 through $p$ happens according to the modal duty cycle (duration) $d_{i}$. Then,

$$
D=\left[d_{1}, d_{2}, \ldots, d_{p}\right]^{T}
$$

gives the PWM period. The total duration of the modal duty cycles must equal the total PWM period, which is usually normalized to unity, i.e., $\|D\|_{1}=1$. If the system has dynamics $y^{(n)}=f_{i}$ when in mode $i$, where $n$ is the system order, and we collect the system dynamics for the $p$ modes in the vector

$$
F=\left[f_{1}, f_{2}, \ldots, f_{p}\right]^{T}
$$

Then a time-averaged model $y_{a}^{(n)}$ of the system dynamics $y^{(n)}$ can be given by [11]

$$
y_{a}^{(n)}=F^{T} D
$$

Therefore, if we can implement the PWM period as a function of a single continuous input $u$, we obtain two things: a duty cycle mapping for the $p$-mode system that can be used to operate the PWM, and a time-averaged dynamic model which can be utilized to design the sliding control.

\section{A. Duty Cycle Mapping for the 3-Mode System}

Shen et al. have applied such a nonlinear model averaging to a 3-mode pneumatic actuator [11]. We will first derive that averaged model and then extend the method to the case of a 
7-mode actuator model. Using (3) for a pneumatic actuator without external disturbances, we have

$$
f_{i}= \begin{cases}f & , \mathrm{i}=1 \\ f+(-1)^{i} b_{i} & , \text { otherwise }\end{cases}
$$

for $i \in\{1,6,7\}$ because, as discussed previously, these are the three modes corresponding to "Close and Close" (mode 1), "Push and Pull" (mode 6), and "Pull and Push" (mode 7) used in [11]. For accommodating a wide range of desired accelerations for the piston of the pneumatic actuator in the positive direction, we would like to be able to appropriately mix modes 1 and 6. Similarly, for creating a wide range of desired piston accelerations in the negative direction, we need to appropriately mix modes 1 and 7. To this end, within each of the positive and negative actuation regions, we select a duty cycle based switching scheme that alternates between no actuation (mode 1) and full action (modes 6 and 7 in the positive and negative directions, respectively). Such a switching scheme is shown in Table II where

$$
d(u)=\frac{u_{H}-u}{u_{H}-u_{L}}
$$

with $u_{L} \leq u \leq u_{H}$. Also, $u=0$ corresponds to no actuation. Substituting (7) and the duty cycles $d_{1}, d_{6}$ and $d_{7}$ listed in Table II into (6), the average system model can be described by

$$
\dddot{y}_{a}= \begin{cases}f+b_{6} u & , \text { if } u \geq 0 \\ f+b_{7} u & , \text { if } u<0\end{cases}
$$

TABLE II

THE 3-MODE DUTY CYCLE MAPPING PROFILE.

\begin{tabular}{|c|c|c|c|}
\hline Region & $u_{L}$ & $u_{H}$ & Duty Cycles \\
\hline- & -1 & 0 & $d_{7}=d(u), d_{1}=1-d(u)$ \\
+ & 0 & 1 & $d_{1}=d(u), d_{6}=1-d(u)$ \\
\hline
\end{tabular}

The scheme for switching between modes 1 and 6 or modes 1 and 7 according to the duty cycles listed in Table II is illustrated in Figure 3(a) as a function of the input $u$. Here, piecewise linear functions changing between 0 and 1 provide the duration for operating different mode. At any given input $u$ level, the duty cycle for high-frequency switching between the two modes present at that input level is determined by the durations $d_{i}$ for the two modes. Figure 2(a) shows (9) in the plane of $\dddot{y}_{a}-f$ versus $u$.

\section{B. Duty Cycle Mapping for the 5-Mode System}

If we extend the accepted values for the index $i$ in (7) to be $i \in\{1,2,5,6,7\}$, the system will include two more control options: "Push and Close" (mode 2) and "Close and Push" (mode 5). This mapping will result in a 5-mode system.

For a 5-mode system, similar to the 3-mode system, we need to decide the scheme for switching between modes. This mode selection scheme involves a new mapping of the single input $u$ to the duty cycle vector $D$. A desirable mapping would utilize at most two modes in any PWM period to simplify the mapping and also minimize the valves' switching for reduced noise and extended lifespan of the valves. Based on (7) and because $b_{i}$ in (3) are all positive, we can see that the open-loop modes can be ordered in terms of the magnitude of the resulting $\dddot{y}$ for each mode as

$$
f_{7} \leq f_{5} \leq f_{1} \leq f_{2} \leq f_{6}
$$

Given this order of actuation level for each mode, it is beneficial to arrange the duty cycles as shown in Table III. Utilizing these mappings, the output $\dddot{y}_{a}$ is increasing (decreasing) with increasing (decreasing) $u$, only two modes are used at a time, and $\|D\|_{1}=1$. The mapping from Table III is plotted in Figure 3(b). Figure 3(b) has four regions matching the same regions as in Table III.

To properly select the values of the transition points $\gamma_{2}$ and $\gamma_{5}$ in Table III, we need to consider Figure 2(b). At $u=0$, mode 1 is utilized $100 \%$ of the time. As $u$ decreases into the negative values (region 2 in Table III), mode 5 is utilized increasingly until $u=-\gamma_{5}$, at which point mode 5 is utilized $100 \%$ of the time. As $u$ further decreases (region 1), mode 5 is used increasingly less, and mode 7 is utilized increasingly until $u=-1$, at which point mode 7 is utilized $100 \%$. The same holds for the positive range of $u$ corresponding to regions 3 and 4 in Table III. Now, if we select the following values for the transition points $\gamma_{2}$ and $\gamma_{5}$

$$
\gamma_{2}=\frac{b_{2}}{b_{6}} \quad \gamma_{5}=\frac{b_{5}}{b_{7}}
$$

then we can see from Figure 2(b) that the resulting averaged dynamics will demonstrate a straight line across the transition points. It should be noted that any other selection of $\gamma_{2}$ and $\gamma_{5}$ will lead to a non-uniformity in terms of actuation, which will not be beneficial to the controller design. Note that since $b_{i}$ in (3) are time-varying functions, $\gamma_{2}$ and $\gamma_{5}$ will also be functions of time.

Straightforward calculations show that the derivation for the 5-mode system leads to a time-averaged model that is the same as that for the 3-mode system. This is distinctly advantageous because we will be able to use the same control signal $u$ for both 3-mode and 5-mode systems. In this case, the only difference between 3-mode and 5-mode operation will be in the resulting valve open/close activity for a given $u$.

\section{Duty Cycle Mapping for the 7-Mode System}

Extending the accepted values for the index $i$ in (7) to $i \in\{1, \ldots, 7\}$, the system will include two more control options: "Pull and Close" (mode 4) and "Close and Pull" (mode 3). This mapping will result in a 7-mode system.

For a 7-mode system, similar to the 5-mode system, a desirable mapping would utilize at most two modes in any PWM period. To properly evaluate the averaged model of the system, we will consider the 7-mode mapping through two separate mappings: The pressurizing profile (which utilizes 
modes $M_{7}, M_{5}, M_{1}, M_{2}$, and $M_{6}$ ) and the venting profile (which utilizes modes $M_{7}, M_{3}, M_{1}, M_{4}$, and $M_{6}$ ). Note that the mode selection scheme in the pressurizing profile in the 7 -mode case is the same as that in the 5-mode case. Based on (7) and because $b_{i}$ in (3) are all positive, we can see that these modes can be ordered in terms of the magnitude of the resulting $\dddot{y}$ for each mode as

$$
\begin{aligned}
\text { Pressurizing Profile: } & f_{7} \leq f_{5} \leq f_{1} \leq f_{2} \leq f_{6} \\
\text { Venting Profile: } & f_{7} \leq f_{3} \leq f_{1} \leq f_{4} \leq f_{6}
\end{aligned}
$$

Given this order of actuation for each mode, it is beneficial to arrange the duty cycles as shown in Table III for the pressurizing profile and Table III for the venting profile. Observe that for the pressurizing profile of the 7-mode system, we are utilizing the same modes and mapping as the 5-mode system in the previous section.

TABLE III

THE 7-MODE DUTY CYCLE MAPPING FOR PRESSURIZING AND VENTING PROFILE.

\begin{tabular}{|c|c|c|c|}
\hline Region & $u_{L}$ & $u_{H}$ & Duty Cycles \\
\hline 1 & -1 & $-\gamma_{5}$ & $d_{7}=d(u), d_{5}=1-d(u)$ \\
2 & $-\gamma_{5}$ & 0 & $d_{5}=d(u), d_{1}=1-d(u)$ \\
3 & 0 & $\gamma_{2}$ & $d_{1}=d(u), d_{2}=1-d(u)$ \\
4 & $\gamma_{2}$ & 1 & $d_{2}=d(u), d_{6}=1-d(u)$ \\
\hline \hline 5 & -1 & $-\gamma_{3}$ & $d_{7}=d(u), d_{3}=1-d(u)$ \\
6 & $-\gamma_{3}$ & 0 & $d_{3}=d(u), d_{1}=1-d(u)$ \\
7 & 0 & $\gamma_{4}$ & $d_{1}=d(u), d_{4}=1-d(u)$ \\
8 & $\gamma_{4}$ & 1 & $d_{4}=d(u), d_{6}=1-d(u)$ \\
\hline
\end{tabular}

Utilizing these mappings, the output $\dddot{y}_{a}$ is increasing (decreasing) with increasing (decreasing) $u$, only two modes are used at a time, and the $\|D\|_{1}=1$. The mapping in Table III is plotted in Figure 3(b) and 3(c).

We select the same values for the transition points $\gamma_{2}$ and $\gamma_{5}$ as in 5-mode (see (11)). Applying the same methodology to the venting profile, we select the following values for the transition points $\gamma_{4}$ and $\gamma_{3}$ in the venting profile:

$$
\gamma_{4}=\frac{b_{4}}{b_{6}} \quad \gamma_{3}=\frac{b_{3}}{b_{7}}
$$

The derivation of the time-averaged 7-mode model is shown in the Appendix A. As it can be seen, the timeaveraged model of the 7-mode system is the same as that for the 3-mode and 5-mode systems. So, we can use the same control input $u$ for 3-mode, 5-mode, and 7-mode systems.

\section{Sliding-Mode Control of the Pneumatic ACTUATOR}

Having expressed the multi-mode discrete-input system in the continuous-input form (9), a sliding-mode approach can be applied for position control of the system. Selecting an integral sliding surface as

$$
s_{p}=\left(\frac{d}{d t}+\omega_{p}\right)^{3} \int_{0}^{t} e_{p} d \tau
$$

where $e_{p}=y-y_{d}$ is the position error and $\omega_{p}$ is a positive gain. The switching function $s_{p}$ provides a measure of the
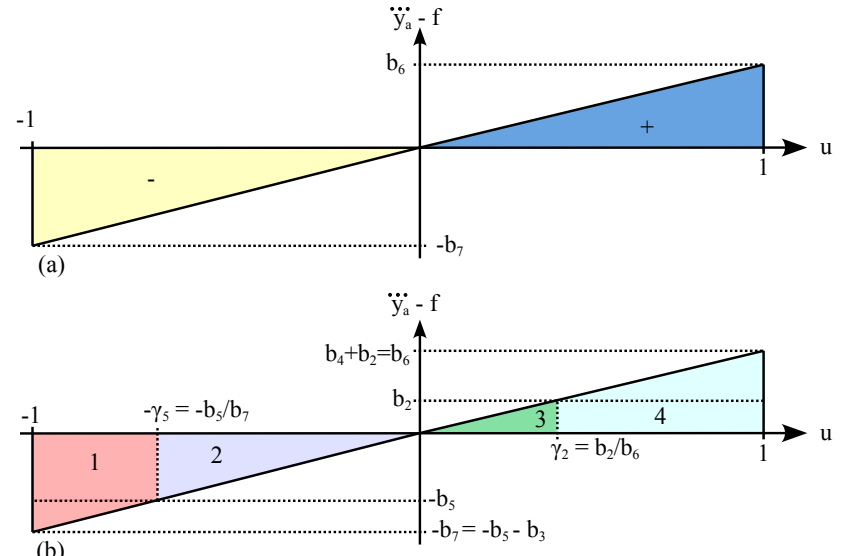

(b)

Fig. 2. Time-averaged input-output relationship for: (a) the 3-mode system, (b) the 5-mode system or 7-mode system (pressurizing profile).

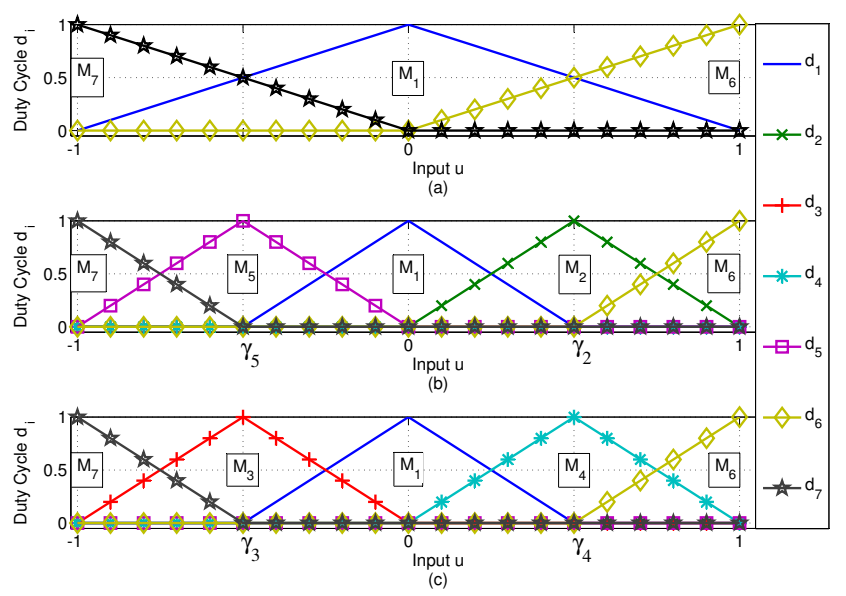

Fig. 3. Duty cycle mapping for: (a) the 3-mode system, (b) the 5-mode system or 7-mode system (pressurizing profile), or (c) the 7-mode system (venting profile).

distance from the sliding surface using the current position error and its derivatives. One can develop a control law based on a sliding-mode approach, in which the equivalent control action, $u_{e q}$, is derived by solving for the input when $\dot{s}_{p}=0$. Taking the derivative of (11) we find

$$
\dot{s}_{p}=\dddot{y}-\dddot{y}_{d}+3 \ddot{e}_{p} \omega_{p}+3 \dot{e}_{p} \omega_{p}^{2}+e_{p} \omega_{p}^{3}
$$

If we substitute (9) as $\dddot{y}$ in (12), we obtain

$$
\dot{s}_{p}=f+\left(b^{+/-}\right) u-\dddot{y}_{d}+3 \ddot{e}_{p} \omega_{p}+3 \dot{e}_{p} \omega_{p}^{2}+e_{p} \omega_{p}^{3}
$$

where

$$
b^{+/-}=\left\{\begin{array}{l}
b_{6}, \text { if } u \geq 0 \\
b_{7}, \text { if } u<0
\end{array}\right.
$$

Solving for $u$ such that $\dot{s}_{p}=0$, we find $u_{e q}$ as

$$
u_{e q}=\frac{\hat{u}}{b^{+/-}}
$$

where

$$
\hat{u}=\dddot{y}_{d}-f-3 \omega_{p} \ddot{e}_{p}-3 \omega_{p}^{2} \dot{e}_{p}-\omega_{p}^{3} e_{p}
$$


Utilizing the control (14) alone does not ensure convergence to the sliding surface in finite time. Also, $\dddot{y}$ of the discrete-input system (actual system) and $\dddot{y}_{a}$ of the continuous-input system (average system) will be somewhat different. To study the robustness of the controller, let us model the actual $\dddot{y}$ as $\dddot{y}_{a}$ (from (9)) plus perturbations. We propose the following theorem where we augment the control action by a robustness term that also ensures convergence to the sliding surface in finite time.

Theorem 1: Consider the perturbed system

$$
\dddot{y}=\left(1+\Delta_{f}\right) f+\left(1+\Delta_{b}\right)\left(b^{+/-}\right) u
$$

where $\left|\Delta_{f}\right| \leq \alpha$ and $\left(\beta_{g m}\right)^{-1} \leq\left(1+\Delta_{b}\right) \leq \beta_{g m}$ (with $\beta_{g m} \geq 1$ ).

The control input

$$
u=\frac{\hat{u}-K \operatorname{sgn}\left(s_{p}\right)}{b^{+/-}}
$$

with the time-variant robustness gain $K$

$$
K=\left(\beta_{g m}\left(\alpha|f|+\eta_{p}\right)+\left(\beta_{g m}-1\right)|\hat{u}|\right.
$$

will ensure convergence to the sliding surface $s_{p}=0$ in finite time where $s_{p}$ is defined in (11).

Proof: To be able to analyze the closed-loop stability, consider the Lyapunov function candidate

$$
V=\frac{1}{2} s_{p}^{2}>0
$$

If $\dot{V}<0$, then $V$ will be decreasing. If $V$ is decreasing, $\left|s_{p}\right|$ will also be decreasing. Assuming $s_{p}$ is initially bounded and $\left|s_{p}\right|$ is decreasing, then $s_{p}$ will be bounded and will asymptotically approach zero. Thus, we intend to control the system so that

$$
\dot{V}=\dot{s}_{p} s_{p} \leq-\eta_{p}\left|s_{p}\right|
$$

Substituting (15), (16), and (17) into (12), we find

$$
\dot{s}_{p}=\left(b^{+/-}\right) u-\hat{u}+\left(\Delta_{f}\right) f+\left(\Delta_{b}\right)\left(b^{+/-}\right) u
$$

Using (17) and (21), we find

$$
\dot{s}_{p}=-K \operatorname{sgn}\left(s_{p}\right)+\left(\Delta_{f}\right) f+\left(\Delta_{b}\right)\left(b^{+/-}\right) u
$$

Substituting (18) into (22), we find

$$
\begin{aligned}
\dot{s}_{p} & =-\operatorname{sgn}\left(s_{p}\right)\left[\beta_{g m}\left(\alpha|f|+\eta_{p}\right)-\operatorname{sgn}\left(s_{p}\right)\left(\Delta_{f}\right) f\right. \\
& \left.+\left(\beta_{g m}-1\right)|\hat{u}|-\operatorname{sgn}\left(s_{p}\right)\left(\Delta_{b}\right)\left(b^{+/-}\right) u\right]
\end{aligned}
$$

Given that $\left(\beta_{g m}-1\right) \geq\left(\Delta_{b}\right)$, and $|\hat{u}| \geq \operatorname{sgn}\left(s_{p}\right)\left(b^{+/-}\right) u$, we find that

$$
\left(\beta_{g m}-1\right)|\hat{u}|-\operatorname{sgn}\left(s_{p}\right)\left(\Delta_{b}\right)\left(b^{+/-}\right) u \geq 0
$$

and thus (23) can be simplified to

$$
\dot{s}_{p} \leq-\operatorname{sgn}\left(s_{p}\right)\left[\beta_{g m}\left(\alpha|f|+\eta_{p}\right)-\operatorname{sgn}\left(s_{p}\right)\left(\Delta_{f}\right) f\right]
$$

Also, given that $\beta_{g m} \geq 1$, and $\alpha \geq \Delta_{f}$, we find

$$
\beta_{g m} \alpha|f|-\operatorname{sgn}\left(s_{p}\right)\left(\Delta_{f}\right) f \geq 0
$$

and thus (25) can be simplified to

$$
\dot{s}_{p} \leq-\beta_{g m} \eta_{p} \operatorname{sgn}\left(s_{p}\right)
$$

Since $\beta_{g m} \geq 1$, we get

$$
\dot{s}_{p} \leq-\eta_{p} \operatorname{sgn}\left(s_{p}\right)
$$

Multiply both sides of (28) by $s_{p}$ and we obtain

$$
\dot{s}_{p} s_{p} \leq-\eta_{p}\left|s_{p}\right|
$$

Thus, the system will converge to the sliding surface $s_{p}=0$ in finite time because of (29).

The above controller leads to the closed-loop stable dynamics

$$
\left(\frac{d}{d t}+\omega_{p}\right)^{3} \int_{0}^{t} e_{p} d \tau=0
$$

in which the position error $e_{p}$ asymptotically tends towards zero.

Utilizing the control action $u$ obtained from (2), (15), (17), and (18), we can apply the closed-loop control to a 3-mode system using the mapping from Table II or to a 7-mode system using the mapping from Table III.

For the 7-mode mapping there are two separate profiles: the venting and pressurizing profiles. The selection between the pressurizing and venting profiles is updated periodically based on the larger output actuation $b_{i}$.

The order of the two modes in any given PWM window was arranged to minimize the overall switching activity, which is the total count of switches made by all 4 solenoid valves divided by the total time. For example, if a PWM window ended with mode $M_{1}$ and the next window contained the $M_{1}$ mode, that mode was used at the start of the next window.

\section{EXPERIMENTAL RESULTS}

\section{A. Experimental Setup}

In this paper, experiments were performed with a 1DOF pneumatic actuator (see Figure 4). The low friction cylinders (Airpel model M16D100D) have a $16 \mathrm{~mm}$ diameter and a $100 \mathrm{~mm}$ stroke. The piston and shaft mass is approximately $\mathrm{M}=900 \mathrm{~g}$. The pneumatic solenoid valves (model GNK821213C3K from Matrix manufacturer) used to control the air flow have switching times of approximately $1.3 \mathrm{~ms}$ (opening time) and $0.2 \mathrm{~ms}$ (closing time). With such fast switching times, the on/off valves are appropriate for the purposes of the proposed control. In terms of sensors, a low-friction linear variable differential transformer (LVDT) is connected to the cylinder in order to measure the linear positions. The controller is implemented using a dSPACE board (DS1104), running at a sampling rate of $500 \mathrm{~Hz}$. This sampling rate has been chosen according to the open/close bandwidth of the valves and to enable an acceptable tracking response. The PWM period was set to $10 \mathrm{~ms}$ and the PWM resolution was 0.2 (5 steps). The experimental setup has the following model parameters: 


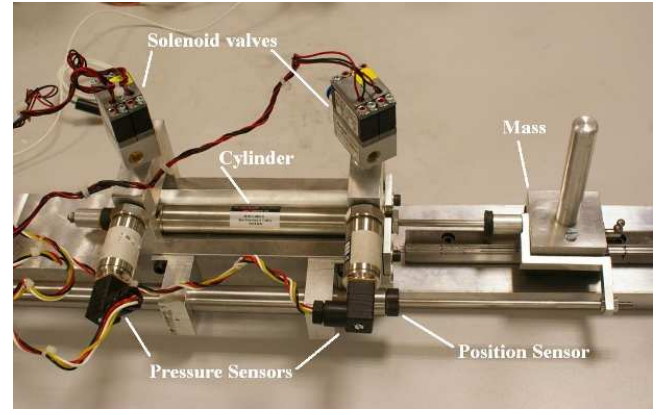

Fig. 4. Experimental setup.

\begin{tabular}{|c|c|c|}
\hline$l$ & $0.1 \mathrm{~m}$ & Chamber Length \\
\hline$T$ & $296 \mathrm{C}$ & Supply Temperature \\
\hline$C_{v a l}$ & $3.4 \times 10^{-9} \mathrm{~kg} /(\mathrm{s} \mathrm{Pa})$ & Mass Flow Rate Const. \\
\hline$P_{S}$ & $300,000 \mathrm{~Pa}$ & Supply Air Pressure \\
\hline$P_{E}$ & $100,000 \mathrm{~Pa}$ & Exhaust Air Pressure \\
\hline & 1.2 & Polytropic Constant \\
\hline$A_{P}, A_{N}$ & $1.814 \mathrm{~cm}^{2}$ & Piston Cylinder Area \\
\hline & $50 \mathrm{~N} \mathrm{~s} / \mathrm{m}$ & Viscosity Coefficient \\
\hline$M$ & $0.9 \mathrm{~kg}$ & Total Mass of load \\
\hline
\end{tabular}

\section{B. 7-Mode Position Control of the Actuator}

This section outlines the experimental testing conducted using a 7-mode controller for the actuator as described in Section IV. The following controller parameters were utilized in (15), (17), and (18): $\omega_{p}=60 \mathrm{rad} / \mathrm{s}, \alpha=0.1, \beta_{g m}=1.1$, and $\eta_{p}=100 \mathrm{~m} / \mathrm{s}^{3}$. The following sine wave test pattern was used to test the position tracking performance of the proposed algorithm:

$$
y_{d}=0.02 \sin (2 \pi f t)
$$

The frequency was varied from $0.1 \mathrm{~Hz}$ to $3.0 \mathrm{~Hz}$. The tracking performance and switching activity was evaluated for this test pattern over a 10 second period.

The results for different sine wave frequencies are plotted in Figure 5(a). From these results, we find that for both the 3mode and the 7-mode systems, increasing the input frequency increases the RMS tracking error. When we compare the results for the 3-mode controller and the 7-mode controller, we can see that for the latter there are notable decreases in both the position error and the switching activity.

We also performed step response experiments to analyze the transient modes (Figure 6). We can remark that the 7-mode controller provides a better tracking error on step changes. To test the system's ability to reject external force disturbance, the experiment was run again utilizing the sinewave test input with a weight attached to the actuator via a cord and pulley. The weights tested were $0.5 \mathrm{~kg}$ (Figure 5(b)) and $1.0 \mathrm{~kg}$ (Figure 5(c)). These weights applied a constant gravity force in the positive direction of the actuator. To prevent the weights attached to the actuator from pendulumlike swinging, only frequencies from $0.1 \mathrm{~Hz}$ to $1.5 \mathrm{~Hz}$ were tested. As the results show, position error was not significantly increased as a result of attaching the weights, and valve switching activity was increased only marginally. This demonstrates the robustness of the controller to external force disturbances.
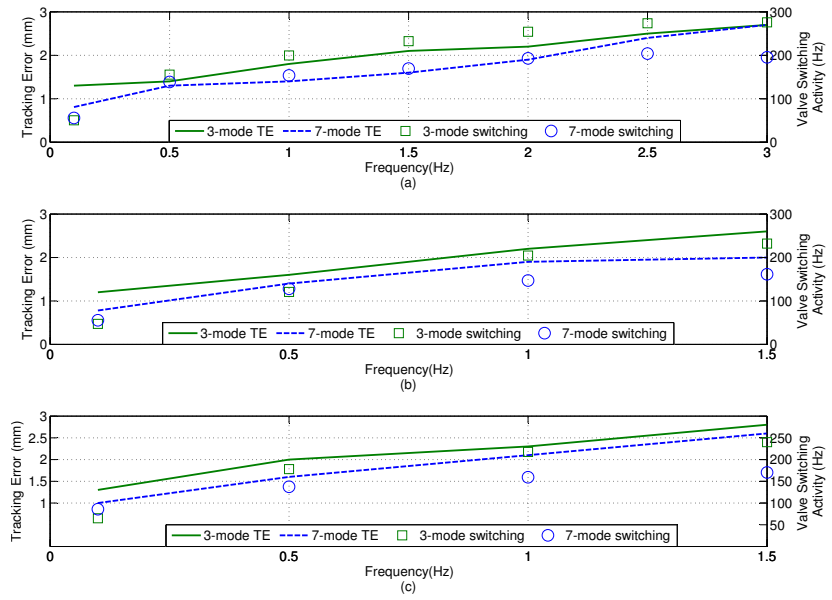

Fig. 5. PWM tracking and switching performance of the actuator with a sine wave input for: (a) No Load on Pulley, (b) $0.5 \mathrm{~kg}$ on Pulley, or (c) 1.0 $\mathrm{kg}$ on Pulley.
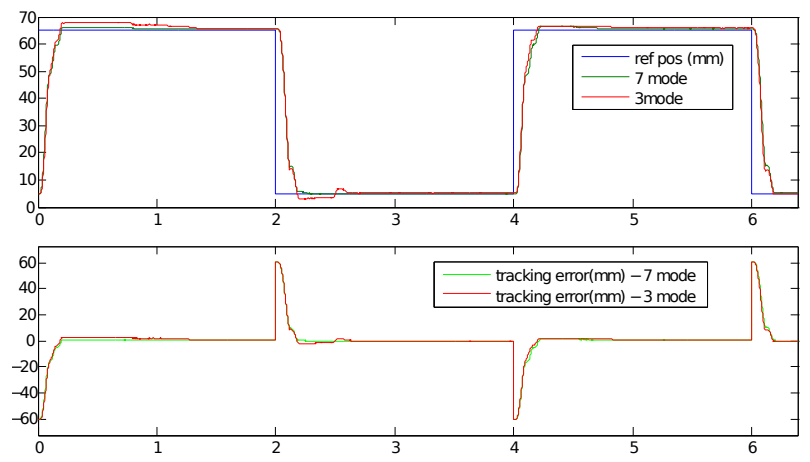

Fig. 6. PWM tracking of the actuator for a square input.

\section{CONCLUDING REMARKS}

In this paper we present the discontinuous system of a pneumatic actuator with solenoid valves. The system under actuation switches between seven modes of operation of the open-loop system. A lot of approaches already could be used to control the discontinuous-time dynamics of such a system and the method proposed in this paper is based on a 7-mode mapping for the PWM to obtain a continuous input time-averaged dynamic model. The averaged model is obtained by averaging the nonlinear open-loop dynamics of the discontinuous system undergoing PWM at the input. This time-averaged model was utilized to create a sliding control law for position control of a pneumatic actuator by varying the PWM duty cycle as a continuous input. This point fundamentally differs from other control techniques on the same type of solenoid valves. This sliding control law selects mode duty cycles for sufficient amounts of drive energy to achieve position tracking. For this sliding control, we developed a stability proof demonstrating convergence of the sliding surface in finite time (and thus asymptotic convergence of the position error).

The 7-mode control was compared experimentally against the 3-mode control. There was a $20 \%$ improvement in tracking performance and $30 \%$ reduction in valve switch- 
ing activity for different situations: a load variation and several sinusoidal inputs. Thus, the performance was found to improve with the additional modes of actuation. These additional modes of operation provided by the 7-mode controller allowed for reduced, yet appropriate amounts of drive actuation. The result was more efficient actuator control.

\section{APPENDIX}

The time-averaged model for the 7-mode PWM controller is derived by evaluating the combination of system dynamics. As noted in the paper, modes $M_{8}$ and $M_{9}$ are not utilized. Denoting the duty cycle vector and the modal system dynamics vector as

$$
\begin{aligned}
D & =\left[\begin{array}{lllllll}
d_{1} & d_{2} & d_{3} & d_{4} & d_{5} & d_{6} & d_{7}
\end{array}\right]^{T} \\
F & =\left[\begin{array}{lllllll}
f_{1} & f_{2} & f_{3} & f_{4} & f_{5} & f_{6} & f_{7}
\end{array}\right]^{T}
\end{aligned}
$$

where

$$
f_{i}= \begin{cases}f & , \mathrm{i}=1 \\ f+(-1)^{i} b_{i} & , \text { otherwise }\end{cases}
$$

for $1 \geq i \geq 7$, the average system dynamics are given by (6); with (A3), we find

$$
\dddot{y}_{a}=F^{T} D=\sum_{i=1}^{7} f_{i} d_{i}=f+\sum_{i=2}^{7}(-1)^{i} b_{i} d_{i}
$$

Please note this model does not consider external disturbances for model based control because these are assumed to be unknown.

Defining the duty cycle vector as given by Table III we can evaluate the time-averaged model for the four regions for the pressurizing profile. To do this we evaluate the time-averaged models partial derivative with respect to the continuous input $u$

$$
\frac{\partial\left(\dddot{y}_{a}\right)}{\partial u}=\sum_{i=2}^{7}(-1)^{i} b_{i} \frac{\partial d_{i}}{\partial u}
$$

The $d_{i}$ are defined by (8). Taking the derivative of (8) we find the slope to be

$$
-\frac{\partial d(u)}{\partial u}=\frac{\partial(1-d(u))}{\partial u}=\frac{1}{u_{H}-u_{L}}=m_{d}
$$

Using these equations the derivatives for $\partial d_{i} / \partial u$ can be found for the four regions in the pressurizing profile.

For region $1 d_{i}=0$ for $i \neq\{5,7\}$. Therefore, for this region the system dynamics are given by

$$
\begin{aligned}
\frac{\partial\left(\dddot{y}_{a}\right)}{\partial u} & =-b_{7} \frac{\partial d_{7}}{\partial u}+-b_{5} \frac{\partial d_{5}}{\partial u}=-b_{7}\left(-\frac{b_{7}}{b_{3}}\right)+-b_{5}\left(\frac{b_{7}}{b_{3}}\right) \\
& =\left(b_{7}-b_{5}\right) \frac{b_{7}}{b_{3}}=b_{3} \frac{b_{7}}{b_{3}}=b_{7} \quad \text { (A7) }
\end{aligned}
$$

Repeating this process for the other 3 regions we can find the time-averaged model's partial derivative with respect to the continuous input $u$.

Thus if we combine the previous results and integrate then, we find the following time-averaged model:

$$
\int_{0}^{u} \frac{\partial\left(\dddot{y}_{a}\right)}{\partial u} d u= \begin{cases}b_{6} u+C & , \text { if } u \geq 0 \\ b_{7} u+C & , \text { if } u<0\end{cases}
$$

Evaluating $C=\left.\dddot{y}_{a}\right|_{u=0}$ we find that $C=f$. Substituting, we find the pressurizing time-averaged model to be:

$$
\dddot{y}_{a}= \begin{cases}f+b_{6} u & , \text { if } u \geq 0 \\ f+b_{7} u & , \text { if } u<0\end{cases}
$$

Repeating the process using the duty cycle vector as given by Table III, we find the time-averaged model for the venting profile is the same as the pressurizing profile.

Comparing (9) and (A9) we find they are the same. Since they have the same time-averaged model they can use the same sliding surface switching function, $s_{p}$, see (11), the same robustness gain, $K$, see (18), and thus the same continuous closed-loop control, $u$, see (17).

\section{REFERENCES}

[1] J. A. Rosas-Flores, J. A. Flores-Campos, and L. G. Corona-Ramírez, "Optimal linearization of the dynamic behavior of an on/off actuated single pneumatic cylinder," in Proceedings of 2008 5th International Conference on Electrical Engineering, Computing Science and Automatic Control (CCE 2008), Mexico City, November 2008, pp. 380385.

[2] A. Girin, F. Plestan, X. Brun, and A. Glumineau, "High-order slidingmode controllers of an electropneumatic actuator: Application to an aeronautic benchmark," International Journal of Control, vol. 79, no. 2, pp. 119-131, 2006.

[3] K. Xing, J. Huang, Y. Wang, J. Wu, Q. Xu, and J. He, "Tracking control of pneumatic artificial muscle actuators based on sliding mode and non-linear disturbance observer," IET Control Theory and Applications, vol. 4, no. 10, pp. 2058-2070, 2010.

[4] A. Messina, N. I. Giannoccaro, and A. Gentile, "Experimenting and modelling the dynamics of pneumatic actuators controlled by the pulse width modulation (PWM) technique," Mechatronics, vol. 15, no. 7, pp. 859-881, 2005.

[5] K. Ahn and S. Yokota, "Intelligent switching control of pneumatic actuator using on/off solenoid valves," Mechatronics, vol. 15, no. 6 pp. $683-702,2005$.

[6] M.-C. Shih and M.-A. Ma, "Position control of a pneumatic cylinder using fuzzy PWM control method," Mechatronics, vol. 8, no. 3, pp. 241-253, 1998.

[7] M. Taghizadeh, A. Ghaffari, and F. Najafi, "Improving dynamic performances of pwm-driven servo-pneumatic systems via a novel pneumatic circuit," ISA Transactions, vol. 48, no. 4, pp. 512-518, 2009.

[8] T. Nguyen, J. Leavitt, F. Jabbari, and J. E. Bobrow, "Accurate slidingmode control of pneumatic systems using low-cost solenoid valves," IEEE/ASME Transactions on Mechatronics, vol. 12, no. 2, pp. 216219, 2007.

[9] V. I. Utkin, "Sliding mode control design principles and applications to electric drives," IEEE Transactions on Industrial Electronics, vol. 40, no. 1 , pp. 23-36, 1993.

[10] V. I. Utkin and H.-C. Chang, "Sliding mode control on electromechanical systems," Mathematical Problems in Engineering, vol. 8, no. 4-5, pp. 451-473, 2002.

[11] X. Shen, J. Zhang, E. J. Barth, and M. Goldfarb, "Nonlinear modelbased control of pulse width modulated pneumatic servo systems," Journal of Dynamic Systems, Measurement, and Control, vol. 128, pp. 663-669, 2006.

[12] S. Hodgson, M. Q. Le, M. Tavakoli, and M. T. Pham, "Improved tracking and switching performance of an electro-pneumatic positioning system," Mechatronics, vol. 22, no. 1, pp. $1-12,2012$.

[13] M.-Q. Le, M. T. Pham, M. Tavakoli, and R. Moreau, "Development of a hybrid control for a pneumatic teleoperation system using on/off solenoid valves," in Proceedings of the IEEE/RSJ International Conference on Intelligent Robots and Systems, Taipei, Taiwan, October 2010, pp. 5818-5823. 